\title{
Study on the Rapid Excavation Technology of Deep Large Cross-section Rock Tunnel
}

\author{
Liu Xinjie, Kong Dezhong, Song Gaofeng \\ Faculty of Resources and Safety Engineering, China University of Mining and \\ Technology(Beijing), Beijing, 100083, China
}

\begin{abstract}
Based on the characteristics of the deep large cross-section rock tunnel, inclined empty wedgy cutting technique, mechanized working line composing of full-hydraulic excavating drill-camel and loader buckets, secondary bolt/shotcreting support technique are proposed. These techniques effectively solve the problems of small footage in the rock tunnel, slow transferring rate and the unstable support in the deep tunnel. The shaping quality is improved; the time of working procedure is shortened and the driving speed is boosted.
\end{abstract}

Keywords: medium deep hole blasting, mechanized working line, secondary support technique

\section{Introduction}

Main text Underground mining dominates the coal production in China. Rock tunnel construction is significant procedure during period of the new mine construction and the tunnel developing in producing mines. Recently, with the development of the fully mechanized equipments and techniques, the production rate has been improved remarkably. The working face that product million or more than ten million tons of coal is not uncommon, which increases the work of tunneling. To meet the requirement of full-rate production, improving the shaping quality and accelerating the tunnel driving are of great importance for the coal industry.

Tunnel excavation is a complicated working procedure which is composed of blasting, loading and transporting and supporting [1-2]. Based on these three key techniques, domestic scholars have conducted a large number of researches [3-5]. From the blasting prospective, empty wedgy cutting technique is proposed in the medium deep hole, which achieve one charge per blasting in the large cross-section rock tunnel. The time of transporting and supporting is significantly shortened by the mechanized driving equipment. With the increasing of mining depth, the conditions of the surrounding rock are changing. According to the flow characteristic, new support techniques which could enforce the stability of tunnels are proposed.

\section{Medium deep hole blasting technique}

Blasting is the major methodology of tunnel driving in China. With the development of heading machine, highpower tunneling equipments appear, which facilitates the application and development of medium deep hole blasting technique. By applying this technique, excavation footage cycle is increased. On the other hand, the burst 
rock quantity per blasting is increased and the time aided working procedure is decreased, which boost the speed and efficiency of driving.

\subsection{Cutting blasting}

The function of cutting is to cave the rock in the working face and create a free surface for the following blasting. Therefore, underhole blasting is the key point of tunnel excavating.

\subsubsection{Cutting method}

Usually, the ration of crater radius $r$ in minimum burden $\mathrm{w}$ is defined as the blasting index.

$$
n=\frac{r}{w}
$$

Parameters : $r$-crater radius;

$w$-minimum burden; $n$-blasting index.

When the $n=1$, the blasting funnel is called normal cast blasting crater; when $1<\mathrm{n}<3$, it is called intensive cast blasting crater; when $0.75<\mathrm{n}<1$ it is called subdued blasting crater and when $\mathrm{n}<0.75$ the blasting could not emerge. The blasting index can be $0.8 \sim 1.0$ during the tunnel excavating and it can be larger during cutting.

The cutting blasting is approximately equivalent to underpart free surface crater blasting under the condition of concentrated charge at intervals. The space between holes is confirmed by the size of plastic zone. The comparison between straight cutting and inclined cutting is shown in the following Figure.1.

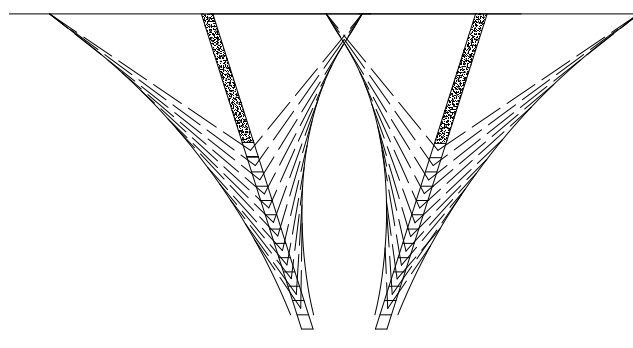

(a) Inclined cutting

Fig.1 Blasting effect

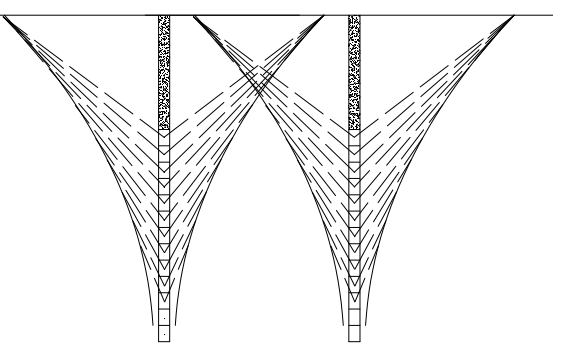

(b) Straight cutting
As can be seen from Figure1, inclined cutting blasting aggravate the rock crush, especially in the large cross-section rock tunnel and it is almost not limited by the width of the section. Therefore, inclined cutting has better application than the straight cutting in the large cross-section rock tunnel driving.

2.1.2 The mechanism and effect of central empty hole

The cutting of central empty hole could induce cracks in the surrounding rock, which will alter the direction of the minimum burden and the direction of blasting effect and create attached free surface for the cut working. According to the free surface hypothesis of rock busting mechanism and stress wave interference hypothesis, the stress wave triggered by underhole blasting could radiate at the free surface and induce tensile failure zone heading to the blasting cartridge. Because extrusion crush is generated by columnar charge blasting and it need little energy to cast the cracked rock in the holes. And the central empty holes can enhance the rock casting and prepare for the following blasting. 
Taking advantage of the hole space and wedgy cutting technique, the central empty hole technique that facilitates the casting rock and deepen the effective depth of the underholes improves blasting efficiency. At the same time, the central empty holes could orient for the blasting and promote the cracked rock.

\subsubsection{Calculation of the charge length}

The cutting blasting is approximately equivalent to underpart free surface crater blasting under the condition of concentrated charge at intervals. The quantity of concentrated charge can be calculated according to the following $\mathrm{M}$. M. Bopeckob formula:

$$
Q=K W^{3}\left(0.4+0.6 n^{3}\right)
$$

Parameters: $Q$ - quantity of concentrated charge(kg);

$K$ - quality index of charge $(\mathrm{kg} \cdot \mathrm{m}-3)$;

$n$-blasting index;

$W$-concentrated charge burden distance (m)

\subsection{Assistant and peripheral holes}

Holes in the roof, floor and the later wall are included by peripheral holes and they are the blastholes for the construction of tunnel section design. They are often fixed right on the outline. The holes in the roof and the later wall are designed to be parallel and the holes in the floor are in the same level. Assistant holes are located between the cutting holes and the peripheral holes and evenly arranged at the intervals of $600 \mathrm{~mm}$. The depth of assistant and peripheral holes is almost the same and the intervals should be controlled relatively small. The low density and low detonation velocity explosives are selected to control the shaping quality and decrease the surrounding rock failure.

2.3 Charge structure and detonation

Charge structure and detonation method are the key points to the blasting result. To guarantee the blasting effect, continuous, decoupling and reverse structure charging are applied in the tunnel excavating. Before charging process, the rock power and water should be cleaned. Millisecond delay detonators are applied for the disposable whole section blasting.

\section{Mechanized working line composing of full-hydraulic excavating drill-camel and loader buckets}

By now, borehole-blasting method dominates the tunnel excavation in China. Boom-type roadheader is still rare in home. There are two types of mechanized working lines based on borehole-blasting method: (1) air-leg rock drill with bucket mechanical mucker or rake mucker; (2) all-hydraulic rock-drilling jumbo with side discharge loader.

The former is mainly used in small section tunnel of small coal mine and its heading speed is relatively. The latter is common in the large section rock tunnel in China. Because the side discharge loader needs to move to and from the reversed loader or conveyors, the distance and time could not be controlled very much and it is not so efficient. Electric full hydraulic pressure drive is applied in the crawler-type bucket loader whose conveyor could load the cracked rock into the loader, which can make the working procedure more efficiently.

\section{Secondary bolt and shotcreting support technique}

Based on the bearing capacity of the surrounding rock, the non-deformability could be enhanced by the shotcreting support technique which makes the surrounding rock become part of the supporting system and control the deformation after excavation. It has several obvious advantages: rapid construction, high mechanization and low cost and is applied widely in the tunnel excavation. 
Traditional disposable bolt and shotcreting support technique mainly enhance the compressive strength of the tunnel surrounding rock. With the increasing of the mining depth, the properties of the surrounding rock will be changed and the rheological behavior will be increasingly obvious. The disposable support focusing on the compressive strength is not effective for the tunnel deformation control any more. Therefore, according to the rheological behavior in the deep and the supporting thesis, the secondary bolt and shotcreting support technique is proposed.

3.1 The mechanism of secondary bolt and shotcreting support technique

In the mechanical equilibrium system of "support-surrounding rock", the roof separation, plastic zone developing and continuous deformation can be partly controlled. It could only undertake a small part of the The compressive deformation of the supporting depends on the motion state of upper rock layer, After the excavation procedure, there emerge obvious displacements towards the tunnel space, especially in the deep surrounding rock. The disposal supporting limits the deformation of the surrounding rock to some degree. The secondary support is conducted after the deformation energy is released.

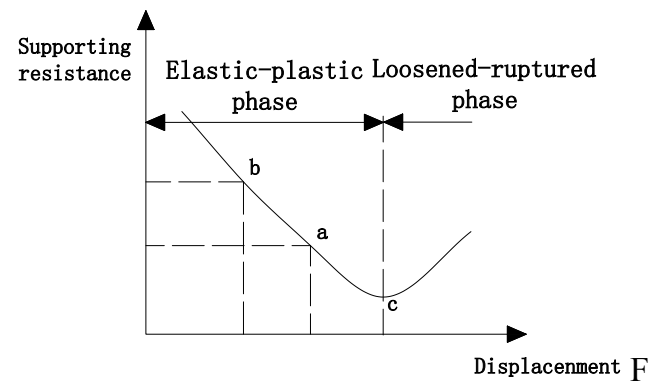

Fig.2 Surrounding rock displacement characteristic
3.2 Optimal supporting time

The secondary support should be conducted at a prosper time that the surrounding rock deformation tends towards stable. According to the soft rock tunnel supporting theory, the optimal supporting time should be controlled at the time when the supporting force of the surrounding rock and the engineering force research the summit.

In the engineering project, the shrinkage distortions of the tunnels are often monitored. The curve of displacements with time can be plotted according to the monitoring data. The inflection point of the curve is often seen as the right time for supporting.

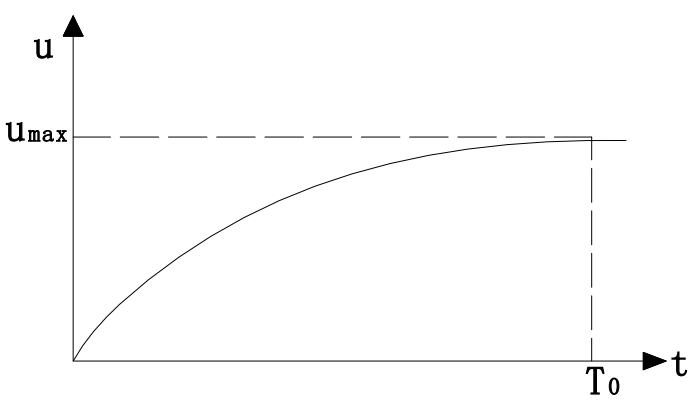

Fig. 3 The optimal supporting time

\section{Conclusions}

(1) The slow footage driving cycle can be effectively controlled by the medium deep hole blasting technique and the layout of the central empty holes can facilitate the rock loading and transporting.

(2) Mechanized working line composing of full-hydraulic excavating drill-camel and loader buckets is applied in the tunnel excavating and promote the heading speed.

(3) Deep rock tunnel deformation could be effectively controlled by the secondary bolt and shotcreting support technique and the supporting time is of significant importance for the tunnel stability. 


\section{References}

[1] Huo X L, Chen S G, Zhang X M, "Study on Construction Characteristic and Temporal-spatial Effect of Tangjiashan Tunnel", CONSTRUCTION TECHNOLOGY, pp 79-83, 2012.

[2] Zuo Q J, "Study on Mechanical Effect of Surrounding Rock for Super-large Cross Section Soft Slate Tunnel during Construction Period", China University of Geosciences, 2013.

[3] Sun X M, Cai F, Yang J, Hong W S, "Numerical simulation of the effect of coupling support of bolt-mesh-anchor in deep tunnel", Mining Science and Technology, pp 352-359, 2009.

[4] HENNING J. G, MITRI H. S, "Examination of hanging-wall stability in a weak rock mass", Metallurgy and Petroleum, Montréal, pp 40-44, 1999.

[5] He M C, Duan Q W, Sun X M, "Computer numerical simulation of soft rock in China" Computer Applications in the Minerals Industries, pp 697-700, 2001. 